\title{
Solar Flares and Coronal Mass Ejections: A Statistically Determined Flare Flux-CME Mass Correlation
}

\author{
A. N. Aarnio壮, K. G. Stassun ${ }^{1,2}$, \\ W. J. Hughes ${ }^{3}$, S. L. McGregor ${ }^{3}$
}

(C) Springer $\bullet \bullet \bullet \bullet$

\begin{abstract}
In an effort to examine the relationship between flare flux and corresponding CME mass, we temporally and spatially correlate all X-ray flares and CMEs in the LASCO and GOES archives from 1996 to 2006. We cross-reference 6733 CMEs having well-measured masses against $12050 \mathrm{X}$-ray flares having position information as determined from their optical counterparts. For a given flare, we search in time for CMEs which occur 10-80 minutes afterward, and we further require the flare and $\mathrm{CME}$ to occur within $\pm 45^{\circ}$ in position angle on the solar disk. There are $826 \mathrm{CME} /$ flare pairs which fit these criteria. Comparing the flare fluxes with CME masses of these paired events, we find CME mass increases with flare flux, following an approximately log-linear, broken relationship: in the limit of lower flare fluxes, $\log (\mathrm{CME}$ mass $) \propto 0.68 \times \log$ (flare flux $)$, and in the limit of higher flare fluxes, $\log (\mathrm{CME}$ mass $) \propto 0.33 \times \log$ (flare flux $)$. We show that this broken power-law, and in particular the flatter slope at higher flare fluxes, may be due to an observational bias against CMEs associated with the most energetic flares: halo CMEs. Correcting for this bias yields a single power-law relationship of the form $\log (\mathrm{CME}$ mass $) \propto 0.70 \times \log$ (flare flux $)$. This function describes the relationship between CME mass and flare flux over at least 3 dex in flare flux, from $\approx 10^{-7}-10^{-4} \mathrm{~W} \mathrm{~m} \mathrm{~m}^{-2}$.
\end{abstract}

Keywords: Flares, Coronal mass ejections

\footnotetext{
${ }^{\dagger}$ Current address: Department of Astronomy, University of Michigan, 830 Dennison Building, 500 Church Street, Ann Arbor, MI 48109. Email: aarnio@umich.edu

${ }^{1}$ Department of Physics and Astronomy, Vanderbilt University, VU Station B 1807, Nashville, TN 37235, USA email: alicia.n.aarnio@vanderbilt.edu

2 Department of Physics, Fisk University, $100017^{\text {th }}$ Avenue N., Nashville, TN, 37208

${ }^{3}$ Department of Astronomy and Center for Integrated Space

Weather Modeling, Boston University, Boston, MA, USA
} 


\section{Introduction}

The physical connection between solar flares and coronal mass ejections has long been a topic of debate and ongoing research in solar physics. CMEs have been observed to occur in conjunction with flares and eruptive prominences (Munro et al., 1979, Webb and Hundhausen, 1987) and with helmet streamer disruptions (Dryer, 1996). While these phenomena are not causally related, it can be definitively said that both X-ray flares and CMEs involve reconfiguration of complex magnetic topologies within the corona via magnetic reconnection. Indeed, Švestka (2001) asserted that CMEs all share the same cause - magnetic field lines opening - and that the factor governing the properties of the resulting $\mathrm{CME}$ is the magnetic field strength in the region whence the CME originates. Nindos and Andrews (2004) proposed that helicity may be the link between flares and CMEs: CMEs have been observed to facilitate helicity loss, "carrying" high helicity magnetic flux from the Sun (Chen et al., 1997; Wood et al., 1999; Vourlidas et al., 2000); LaBonte, Georgoulis, and Rust (2007) observed that active regions producing $\mathrm{X}$-class flares generate enough helicity to match that lost via CME within hours to days after the flare.

Efforts have been made to identify a physical link between the two phenomena by correlating properties of cospatial, contemporaneous flares and CMEs. Although flares themselves are not the cause of CMEs, their properties could serve as prediction tools for imminent CMEs and particle events, which are of much concern in the field of space weather.

Statistical relationships between solar flares and CMEs are of interest to astronomers as large scale time-series X-ray observations have found solar-like X-ray activity on T Tauri Stars (hereafter referred to as TTS; solar-mass, premain sequence stars). Indeed, Haisch, Antunes, and Schmitt (1995) noted that stellar X-ray flare light curves behave similarly to solar X-ray flares, that is, the structure of the light curve (impulsive initial rise followed by exponential decay) is effectively identical, though the TTS flares are often several orders of magnitude more energetic. Based on these observations, theory and modeling of stellar flares has been guided by the premise that the physics behind both solar and stellar X-ray flares is the same (e.g., Reale et al., 1997). Peres et al. (2001) demonstrated that stellar coronal X-ray emission could be reproduced by "scaling up" solar observations to include greater fractional coverage of the surface by active regions and solar-like coronal structures. Further underscoring the similarities in solar/stellar coronae, features similar to helmet streamers and slingshot prominences have indeed been found on TTS (e.g., Massi et al., 2008, Skelly et al., 2008).

In many applications of TTS X-ray studies, the observable phenomenon is the X-ray flare, but the desired quantity is the associated mass loss. While solar CMEs do not shed large quantities of mass, young Suns $(\approx 1 \mathrm{Myr})$ exhibit $\approx 3$ orders of magnitude more energetic flares at a higher frequency, and the problem of how substantial angular momentum vis-à-vis mass is shed in these stars remains unresolved. Additionally, CME-like events on young stars could aid in understanding circumstellar disk evolution and planet formation: 
for example, could CMEs on young stars be a mechanism for "flash-heated" chondrule formation (Miura and Nakamoto, 2007)?

To be clear, the motivation for this work is not to suggest a causal solar flare-CME relationship. We seek to quantify a relationship between solar flare fluxes and CME masses under the general premise that in some cases, flares and CMEs arise from common regions of complex magnetic topology and high field strength and thus may have correlated properties. Specifically, we aim to calibrate a relationship between solar flare flux and CME mass for the purpose of application to other stars possessing solar-like coronae.

\section{Archival Data}

We obtained archival measurements of flares and CMEs for the period of January 1996 to December 2006. The LASCO CME database (Gopalswamy et al., 2009) catalogs observations of the Large Angle and Spectrometric Coronagraph dating back to January, 1996. LASCO observations were complete for $\approx 83 \%$ of the 11 years analyzed in this work, with 13862 manually identified CMEs and their measured parameters from this period. Of particular interest in this work, 6733 CMEs with well-measured masses - i.e. not halo CMEs or CMEs which faded before entering the LASCO field of view - are reported (see Figure 1), along with their linear speeds, accelerations, angular widths and central position angles (CPAs). CME masses are measured by converting brightness per pixel into mass per pixel; this calculation assumes the observed brightness is light Thomson scattered off of electrons in a mixture of $90 \%$ ionized Hydrogen and $10 \%$ Helium. All pixels within a manually defined CME shape are then integrated to obtain a total CME mass (Vourlidas et al., 2000). The CME start time reported is when the $\mathrm{CME}$ is first detected in the $\mathrm{C} 2$ telescope field of view; $\mathrm{C} 2$ images the region from 2.0 to $6.0 R_{\odot}$.

Spanning more than three decades of observation, the Geostationary Operational Environmental Satellite (GOES) flare databas 2 reports the 1-8 $\AA$ band full-disk X-ray flux at Earth; flare classifications are then applied based upon the peak flux in that bandpass. From 1996-2006, the database contains information for 22674 flares. Of these, positions for optical counterparts are documented for approximately half of the flares in the database (Figure 2). Of these 12050 flares, one A class, 3638 B, 7248 C, 1056 M, and 107 X class flares are recorded.

\section{Determining Flare-CME Association}

As described above, we use the 6733 CMEs from the LASCO CME catalog with well measured masses and the 12050 flares with optical counterpart positions from the GOES flare database.

\footnotetext{
${ }^{1}$ This CME catalog is generated and maintained at the CDAW Data Center by NASA and The Catholic University of America in cooperation with the Naval Research Laboratory. SOHO is a project of international cooperation between ESA and NASA.

${ }^{2}$ http://www.ngdc.noaa.gov/stp/SOLAR/ftpsolarflares.html
} 
In determining whether a given flare and CME are associated, we utilize spatial and temporal criteria, requiring that both flare and CME occur within a set time window and angular separation. We initially set the time window generously to select CMEs which occur within $\pm 2 \mathrm{~h}$ of a flare's start time (though we refine this below). Converting Stonyhurst system flare positions (Cartesian) on the disk to spherical coordinates, we show in Figure 3 the angular separations of these time-matched CMEs and flares. A clear peak is seen about $0^{\circ} \pm \approx 70^{\circ}$; we adopt a separation criterion of $\pm 45^{\circ}$ (e.g., both events occur in the same quadrant of the disk with respect to the $\mathrm{CPA}$ of the $\mathrm{CME}$ ) as a value intermediate to the $\pm 70^{\circ}$ in our Figure 3 and the $\approx \pm 30^{\circ}$ results of Yashiro et al. (2008) . Interestingly, but beyond the aims of this work, we note that there is a significant peak for flares which occur $\approx 180^{\circ}$ in separation from the CME CPA, potentially indicative of large-scale correlated disruptions.

After applying the $\pm 2 \mathrm{~h}$ temporal and a $\pm 45^{\circ}$ angular separation cut, we assess whether the initially chosen, large time window can be narrowed (Figure 4). Regardless of which flare time (start, peak or end) we use, there is a significant peak between 0-80 min in the resulting flare/CME pairs' time separations (CME start time - flare time). Other time separations surrounding these peaks appear to represent a "background" level at $N \approx 60$ pairs per 10 min time bin; these could represent randomly matched flare-CME pairs which are not truly associated. Events appear to be the most strongly correlated when using the flare start time (solid, black histogram in Figure 4); this is to say that the number of events in the 10-80 min bins are greater in number and there is less "background" variation than is seen for the other flare time choices. We therefore adopt the flare start time, but in any case this choice does not strongly affect our final results.

It is important to note that the CME "start time" in fact corresponds not to the time when the CME was launched, but rather to the time the CME first appears in the $\mathrm{C} 2$ detector (at a height of $\approx 3.23 R_{\odot}$ on average). Using the linear speeds of the CMEs reported in the LASCO database, we can calculate the approximate CME travel times from the solar surface. The average travel time so computed is $75 \pm 17 \mathrm{~min}$, corresponding very well to the average time offset between the CME "start times" (detection times) and their associated flare times (Figure 4). Note that this simple calculation assumes negligible acceleration of the CMEs. Thus, while we cannot say with certainty that any one correlated flare/CME pair does not truly have a time delay, the data are broadly consistent with flare/CME start times that are simultaneous at the solar surface.

Interpreting the 10-80 min peak of Figure 4 as a time offset region during which there is a higher probability of finding associated flares and CMEs, we narrow the time correlation window to accept CMEs which occur 10-80 min after a flare (vertical, triple dot-dashed lines, Figure 44). This is consistent with the findings of Andrews and Howard (2001), Mahrous et al. (2009); in the latter work, the analysis used flare peak flux times and end times. This 10-80 min choice in temporal separation could potentially eliminate some true flare-CME pairsprevious studies (e.g., Harrison, 1991, Harrison, 1995) have shown instances in which CMEs precede flares - but we do not consider these cases due to the absence of a distinct peak in the range of negative time offsets. The 10-80 min 
window also evidently includes some "background" events. Potentially, these cases only represent a minority of flare-associated CMEs.

Since we cannot tell from this data set whether a CME originates on the visible side of the limb or just beyond it, it is possible that some "back-sided" CMEs are spuriously associated with flares on the visible side. We can estimate the effect of back-sided CMEs using geometric arguments. First, we assume that CMEs observed by LASCO can be detected if they originate from a $260^{\circ}$ longitudinal range about the Sun (i.e., from the full $180^{\circ}$ of the visible disk, plus $40^{\circ}$ beyond both the eastern and western limbs), and we assume that CMEs are equally probable to originate from anywhere within this area. Thus, $33 \%$ of all CMEs $\left(80^{\circ} / 240^{\circ}\right)$ could be back-sided CMEs that are then potentially paired with flares on the visible solar disk. Our spatial cut requires $\pm 45^{\circ}$ coincidence in polar angle; assuming CMEs are equally likely to occur over all $180^{\circ}$ of polar angle, this serves to eliminate half of all spurious correlations, reducing the number of back-sided CMEs potentially introduced to our correlated sample to $16 \%$. Further reducing this is our time-matching requirement, wherein we select only the CME-flare pairs occurring within $70 \mathrm{~min}$, out of the full 240-min time window searched (i.e., $70 / 240=29 \%$ ). Combining these, we potentially include $5 \%$ of back-sided CMEs in our flare-CME pairs. Of course, some of these backsided CMEs may in fact be truly associated with the observed flares; however, as a conservative statement, back-sided CMEs should represent at most a 5\% contribution of spuriously flare-correlated CMEs in our sample and thus likely add only modestly to the scatter in our correlated quantities.

\section{Results}

\subsection{Frequency of Associated Flares and CMEs}

With each of the above constraints applied, the total number of flare-CME pairs in the sample is winnowed as illustrated in Figure 5. The final sample contains 826 flare-CME pairs, 737 of these are unique. As performed, the correlation allows for the selection of multiple CMEs per flare, and one CME may be found to be associated with multiple flares. With the data available, there is no way to distinguish which CME may "actually" be associated with the flare, or if indeed there should be only one associated. We therefore use all 826 pairs when comparing flare and CME properties, but we use only the 737 unique CMEs' properties when analyzing properties of flare-associated CMEs vs CMEs occurring without flares.

Of the 6733 CMEs we perform the correlation with, 737 unique CMEs are found to be associated with flares; put another way, we find $11 \%$ of CMEs to be flare-associated. Here we assess whether our applied criteria for correlation or observational effects could be decreasing the number of associations found, and in which variables these issues may create the most problems. In particular, the LASCO data gaps in addition to CME width and projection effects likely make our $11 \%$ an underestimate. For comparison, previous estimates of CMEs associated with flares are 40\%, (Munro et al., 1979) and 34\% (St. Cyr and Webb, 1991). 


\subsubsection{Data Gaps}

LASCO coverage was $83 \%$ complete from 1996-2006. We found that the following numbers of flares occurred during a LASCO data gap: $24 \mathrm{X}, 119 \mathrm{M}, 595 \mathrm{C}$, and 803 B class. These flares could have been "lost" to our analysis, as the data gaps ended 10 min or more after the flare, within our 10-80 min temporal selection window. These values do not reflect, however, cases in which a CME did occur after the data gap ended and the flare was paired with that CME; we could not exclude these cases from the analysis as the technique does not allow for discerning which flares and CMEs are "actually" associated. Proportionately, more high energy flares occur during data gaps, likely because the particle events associated with the flares cause gaps in coverage by the LASCO C2 detector. B flares are excluded from this statement, as their energies are near the detection threshold for the GOES detector. An additional caveat to this is that the numbers of "lost" flares reported above do not account for simultaneity of flares; multiple flares could occur in a given data gap (thus, we cannot conclusively link a given class flare to a higher frequency of particle events), and we have counted each singly.

\subsubsection{Wide, Partial Halo, and Halo CMEs}

Surprisingly, we find that in the application of our time and angular separation constraints, most of the $107 \mathrm{X}$ class flares are lost from the sample. Only seven of the 826 pairs have $\mathrm{X}$ class flares. This contradicts the results of Andrews 2003, who found an almost $100 \%$ X-flare-CME association 3 .

A limitation imposed by our data completeness requirement for the flares and CMEs analyzed in this work (see Section 2] we require flares to have positions, and CMEs to have well measured masses) is that we have excluded halo CMEs, as their masses cannot be measured via the same technique used for CMEs perpendicular to our line of sight. Additionally, our analysis excluded CMEs which had high errors in their mass measurements; these CMEs are illustrated in Figure 6 as a red histogram. Most of the CMEs with poorly constrained masses are what would be designated as "wide" or "partial halo" CMEs (i.e., with observed widths greater than $60^{\circ}$ ).

In an effort to account for these selection effects in our analysis, we performed the correlation again, including wide, partial halo, and halo CMEs. This inclusion increased the CME sample size to 8128 CMEs. Because they lack clearly defined CPAs, we did not require spatial coincidence for halo CMEs and flares, thereby including any halo CMEs which were time-correlated with flares. CMEs with poor mass measurements typically do have documented CPAs, so those events were required to be co-spatial with associated flares within $\pm 45^{\circ}$, as previously. This resulted in 1153 flare-CME pairs, with $53 \mathrm{X}, 278 \mathrm{M}, 608 \mathrm{C}$, and $214 \mathrm{~B}$ class flares. Of the 1153 pairs, 1028 of the CMEs are unique, resulting in a 13\% flare-association; this is effectively indistinguishable from the association fraction observed when including only CMEs with good masses (Section 4.1). While the

\footnotetext{
${ }^{3}$ Note, however, that Andrews 2003 set out specifically to define a sample of associated "big
} flares" and CMEs. 
entire sample is increased by $\approx 40 \%$ and we don't find a greater fraction of CMEs to be associated with flares, the greatest percentage increases occur with higher flux flares. Including CMEs with poorly measured masses and halo CMEs results in $\approx 8$ times as many $\mathrm{X}$ class flares, and almost double the $\mathrm{M}$ class flares. $\mathrm{C}$ and $\mathrm{B}$ class flares show only modest $\approx 20 \%$ increases in representation in the sample. We assess below (Section 4.2.4) the main effect this could have on our results, and show a method to possibly correct for it.

In summary, we lose $\approx 75 \%$ of $\mathrm{X}$ class flares to the combination of data gaps and observational selection effects, and this "loss fraction" is greatest for the strongest flares, decreasing with decreasing flare flux. Despite these factors potentially over-narrowing our correlated sample, we have demonstrated that the requisite time and position conditions applied here are sound in reasoning and represent a conservative approach which may only under-count flare-CME pairs.

\subsection{Correlations in the Matched Flare-CME Sample}

For the matched sample of 826 flare-CME pairs (see Section 3), we assess potential relationships between five different flare and CME parameters: flare flux, CME linear speed, CME acceleration, CME width, and CME mass. Primarily, we seek to define a flare flux-CME mass relationship. We also perform additional correlations with our large flare-CME pair sample in order to compare with previous work and to elucidate the underlying physics.

\subsubsection{CME Linear Speed, Acceleration, and Flare Association}

Flare and eruptive prominence-associated CMEs have been observed to have higher linear speeds than those which are not associated with flares (Gosling et al., 1976 Moon et al., 2003). To check whether our flare-CME pairs reflect this observed relationship, we show in Figure 7 the two distributions of CME linear speeds for our sample. The mean linear speed for CMEs associated with flares is $495 \pm 8$ $\mathrm{km} \mathrm{s}^{-1}$; CMEs not associated with flares have an average speed of $422 \pm 3 \mathrm{~km}$ $\mathrm{s}^{-1}$ (the error we report here is the standard deviation of the mean). A $t$-test (Press et al., 1995) assesses the probability that two distributions have significantly different means. The $t$ statistic for these distributions is 8.5 , and the corresponding null-hypothesis probability (that the two distributions have the same mean) is $\ll 10^{-6}$.

Due to the asymmetric skew of the distributions, we verify this result by performing a non-parametric analog of the $t$-test, the Wilcoxon-Mann-Whitney test (also known as a $U$ test). The statistic is $\mathrm{Z}$, and the corresponding probability assesses the likelihood, when sampling two distributions, of preferentially finding a higher value in one distribution than another. The result of the $U$ test corroborates the $t$-test: the $Z$ statistic is -9.0 , and the null-hypothesis probability is $\ll 10^{-6}$. We conclude that these two distributions have statistically significantly different means, consistent with observations that flare-associated CMEs have higher linear speeds than CMEs not associated with flares.

Figure 8 shows the relationship between CME speed and acceleration. Evidently, there is a trend of decreasing acceleration (or rather, increasing deceleration since the mean accelerations are negative) with increasing CME linear 
speed. To test this statistically, we perform a Spearman correlation test, finding the $\rho$ coefficient to be -0.22 - a monotonically decreasing relationship - and the corresponding null-hypothesis probability $\ll 10^{-6}$. In summary, we see that the fastest CMEs are associated with flares, and the fastest CMEs decelerate the most on average.

\subsubsection{CME Mass, Acceleration, Flare Flux}

In Figure 9we examine more closely the potential effect of CME mass on acceleration for CMEs associated with flares. The highest mass division includes CME masses $\geq 10^{15} \mathrm{~g}$, while the middle and lowest mass subsets span the ranges of $10^{14}$ $\mathrm{g} \leq \mathrm{CME}$ mass $<10^{15} \mathrm{~g}$ and CME mass $<10^{14} \mathrm{~g}$, respectively. Again utilizing the $U$ test, we find that all three distributions have statistically indistinguishable means.

In Figure 10, CME acceleration in the flare-associated case and the converse scenario is shown. The mean of the CME-flare pair distribution is $-1.8 \pm 0.1 \mathrm{~m}$ $\mathrm{s}^{-2}$, while the mean of the distribution of CMEs without flares is $0.07 \pm 0.25 \mathrm{~m}$ $\mathrm{s}^{-2}$ (the errors on these quantities are the standard deviations of the mean). We use a $t$-test to determine whether the acceleration distributions have significantly different means. The $t$ statistic is -2.5 , the corresponding null-hypothesis probability 0.01 ; this indicates these distributions do indeed have significantly different means. A $U$ test was also performed; the $Z$ statistic found to be 4.6 , and the null-hypothesis probability is $1.9 \times 10^{-6}$. The CME-flare pairs have CMEs which tend to decelerate on average, while the CMEs not associated with flares are centered about 0 acceleration.

We next assess how the magnitude of this deceleration for flare-associated CMEs may relate to the flux of the associated flare. In Figure 11, the relationship between CME acceleration and flare class is shown for the 826 CME-flare pairs. X and $\mathrm{M}$ flares are combined for this comparison, as there are only $7 \mathrm{X}$ class flares in the 826 flare-CME paired sample. We employ the $U$ test, making no assumption that these distributions are Gaussian. Comparing the mean accelerations of B class and weaker with $\mathrm{C}$ class flare-associated CMEs, the $Z$ statistic is -1.9 , with a corresponding null-hypothesis probability of 0.03 . Applying the $U$ test to intermediate strength flares, the difference in deceleration between $\mathrm{C}, \mathrm{M}$, and X class flare-associated CMEs is not statistically significant. However, testing the same group of B/A class flare-associated CMEs against $\mathrm{M}$ class and stronger flare-associated CMEs, the $Z$ statistic is -2.8 , its null-hypothesis probability 0.002. Evidently, just as flare-associated CMEs decelerate on average, CMEs associated with the strongest flares show the most deceleration.

\subsubsection{CME Width, Mass, and Flare Flux}

We have so far shown above that higher energy flares are associated with faster CMEs that decelerate the most. To test whether higher energy flares are also associated with faster, more massive CMEs, we checked to see if the width of the CME was related to the flux of the associated flare. In Figure 12 we show that indeed, higher flux flares are associated with wider CMEs. With increasing 
flare flux, the width of the associated CME increases: the mean widths (plus or minus the standard devations of the mean widths) of the flare-associated CMEs are $80 \pm 10^{\circ}, 63 \pm 1.8^{\circ}, 53 \pm 0.9^{\circ}$, and $42 \pm 1.4^{\circ}$ for $\mathrm{X}, \mathrm{M}, \mathrm{C}$, and B class flares, respectively.

Considering projection effects, it is unsurprising then that as the mean CME width increases, so too does the occurrence of halo CMEs. Halo CME occurrence is also shown in Figure 12, with $80 \%$ of $\mathrm{X}$ class flares being associated with halo CMEs, while M, C, and B class flares have halo CME associations of $21 \%, 6 \%$, and $3 \%$, respectively. A $t$-test shows the mean widths of CMEs associated with $\mathrm{M}, \mathrm{C}$, and B class flares are significantly different. Comparing the mean widths of CMEs associated with $\mathrm{M}$ and $\mathrm{C}$ class flares, the $t$ statistic is 5.4, its nullhypothesis probability $\ll 10^{-6}$. For the mean widths of CMEs associated with $\mathrm{C}$ and $\mathrm{B}$ class flares, the $t$ statistic is 6.2 , and the corresponding probability is also $\ll 10^{-6}$.

Finally, we find that as CME width increases, so too does the mass of the CME (Figure 13). Put another way, the widest CMEs aren't necessarily more diffuse than the more narrow CMEs.

\subsubsection{CME Mass, Flare Flux}

For the associated flares and CMEs, the relationship between CME mass and flare flux is shown in Figure 14. Dividing the pairs by flare flux into four groups, we fit the resulting distribution with Gaussian functions. It is clear that the Gaussian centroids progress to higher CME mass as the flare flux sampled increases (Figure 14).

In Figure 15, the 826 flare/CME pairs are binned into fourteen equal sets of $N=59$ pairs each. This division was chosen to maximize the number of events with which to perform statistics. Visually, it is apparent that the mean CME mass in each bin increases with flare flux. There is an apparent "knee" to the function around $\log$ (flare flux $) \approx-5.5$, so we use two linear regression fits to describe the functional relationship on both sides of this "knee." The first function is fit to bins $0-8$, and the second to bins $7-13$, counting from left to right. There is slight overlap in that precisely where the "knee" of the function occurs is unclear. The first linear fit is of the form:

$$
\log (\mathrm{CME} \text { mass })=(18.5 \pm 0.57)+(0.68 \pm 0.10) \times \log (\text { flare flux })
$$

The second linear fit follows the form:

$$
\log (\mathrm{CME} \text { mass })=(16.6 \pm 1.30)+(0.33 \pm 0.26) \times \log (\text { flare flux })
$$

We utilize Spearman's $\rho$ correlation test to assess the degree of correlation of flare flux and CME mass. The test does not assume a particular form of correlation, rather, a $\rho$ coefficient of unity indicates complete monotonic relationship of the variables tested. For the mean values in each bin, we find the $\rho$ coefficient to be 0.99 with a null-hypothesis probability of $\ll 10^{-6}$. This $\rho$ coefficient nearly equal to one and the corresponding very low probability confirm that these are highly significantly correlated properties. 
We also performed a Pearson linear correlation test. The Pearson coefficient ranges from -1 to +1 and evaluates how linearly related two parameter sets are. We obtain a value of $r=0.96$ when comparing the mean masses and fluxes in Figure 15. This strong correlation means one could nearly perfectly predict the mean behavior, but do note that there is a scatter about each mean mass of $\approx 1$ dex.

To assess whether a broken fit is indeed necessary, we fit the correlated flux and mass bins of Figure 15 with a single linear fit. The $\chi_{\nu}^{2}$ value of this fit is 0.998 , while the $\chi_{\nu}^{2}$ values for each of the fits above are 0.75 and 0.76 , respectively. We also determine the robustness of the "knee" by re-binning the data and fitting again. The data divided this time into seven bins of $N=118$, we fit a single line. This fit has a $\chi_{\nu}^{2}$ of 0.87 . For comparison, we apply another broken log-linear fit to the data with the "knee" placed at the same point, $\log ($ flare flux $) \approx-5.5$. Each of the functions better fits the data $\left(\chi_{\nu}^{2}\right.$ values of 0.16 and 0.07 , respectively, potentially indicating we have "over-fit" the data). In both cases, we find that fitting the CME mass-flare flux relationship with a broken linear function indeed produces a better fit than simply fitting all of the data points with a single linear function. We also find that the "knee" is robust and does not depend upon how the data are binned.

As mentioned in Section 4.1.2, observational effects could be causing "lost" flare/CME pairs in the highest flare flux regime, which could in turn be causng the knee in the relationship of Figure 15. In Section 4.1.2, we re-introduce wide, partial halo, and halo CMEs to the analysis. Halo CMEs lack mass measurements, but what we do know about halo CMEs allows us to assign an upper limit to possible masses. We have shown that the highest flux flares are most often associated with halo CMEs, and the highest flux flares tend to be associated with the widest CMEs (Figure 12). We have also shown that the widest CMEs are the most massive (Figure 13). Putting this expanded sample into a binned representation as in Figure 15, we obtain a range of CME masses for the given flare flux of the pair, and assign, as an upper bound, the maximum possible mass for that bin to the halo CME. In this way, we obtain an estimate of the greatest possible mean masses per bin when including halo CMEs.

In Figure 16, we reproduce Figure 15, now including halo CMEs and CMEs with poorly constrained masses. The linear fit that describes this relationship is of the form:

$$
\log (\text { CME mass })=(18.67 \pm 0.27)+(0.70 \pm 0.05) \times \log (\text { flare flux }) .
$$

In this case, single power-law fit is sufficient to adequately describe the data. As this case assumes the maximum possible halo CME mass, the true CME mass/flare flux relationship likely lies in the parameter space bounded by the broken fit of Figure 15 and the single fit shown in Figure 16.

\section{Discussion and Conclusions}

For flares and CMEs arising from the same active regions, one would anticipate some of their properties to be correlated. For example, a less magnetically 
complex active region may produce lower flux flares and lower mass CMEs. Conversely, a highly magnetically complex active region may produce both higher energy flares and more massive CMEs.

Our method for pairing flares and CMEs creates a very large data set with which to statistically examine whether flare and CME parameters are correlated. With this data set, we have found correlated properties of associated flares and CMEs which are consistent with previous observations, and we have furthermore shown a relationship between the flux of the flare and the mass of the CME. In summary, we have shown:

1. CMEs associated with flares have higher average linear speeds than CMEs not associated with flares (Section 4.2.1, $495 \pm 8 \mathrm{~km} \mathrm{~s}^{-1}$ and $422 \pm 3 \mathrm{~km} \mathrm{~s}^{-1}$, respectively),

2. CMEs with higher linear speeds tend to decelerate; the relationship of acceleration and linear speed is correlated, and monotonically decreasing (Section 4.2.1),

3. Flare-associated CMEs tend to decelerate: flare associated CMEs have a mean acceleration of $-1.8 \pm 0.1 \mathrm{~m} \mathrm{~s}^{-2}$ while CMEs not associated with flares have a mean acceleration of $0.07 \pm 0.25 \mathrm{~m} \mathrm{~s}^{-2}$ (Section 4.2.2),

4. The magnitude of a flare-associated CME's deceleration increases with increasing flare flux: $\mathrm{M}$ class and stronger flare-associated CMEs have a mean acceleration of $-3.7 \pm 1.3 \mathrm{~m} \mathrm{~s}^{-2}$, while B class and weaker flare-associated CMEs have a mean acceleration of $0.14 \pm 0.8 \mathrm{~m} \mathrm{~s}^{-2}$ (Section 4.2.2),

5. A CME's mass is apparently unrelated to its acceleration (Section 4.2.2),

6. The width of a flare-associated CME is directly correlated with the flux of the flare, with X class flare-associated CMEs being the widest $\left(80^{\circ} \pm 10^{\circ}\right)$ and B class flare-associated CMEs the most narrow $\left(42^{\circ} \pm 1.4^{\circ}\right)$,

7. Wider CMEs are more massive than narrow CMEs (Section 4.2.3, Figure 13), and

8. The stronger the associated flare is, the more massive the CME is (Section 4.2.4); quantitatively, we find $\log (\mathrm{CME}$ mass $) \propto 0.7 \times \log$ (flare flux).

The final bullet point in the list above could have very interesting implications for studies of young, solar-type stars, which exhibit high-frequency, high energy $\mathrm{X}$-ray flaring activity. With the functional relationships illustrated in Figures 15 and 16] it is now possible to extrapolate a CME mass/flare flux function into the range of flare fluxes observed for young stars (hatched region, Figure 15). Whether the second function of the broken log-linear relationship (Figure 15) or the single function of the analysis including halo and poorly measured CME masses (Figure 16) would be used in this extrapolation remains to be seen; additional analyses of halo CME masses will be necessary to determine whether the "knee" exists for physical reasons and remains present when halo CMEs are included with better mass estimates. If the change in slope is physical, it could potentially represent a saturation point, approaching a set limit of possible CME mass. For young stars, it would be interesting to see whether this saturation point has any correlation with the saturation point in X-ray activity. 
Acknowledgements This research is supported by NSF grant AST-0808072 (K. Stassun, PI). K. G. S. gratefully acknowledges a Cottrell Scholar award from the Research Corporation, and a Diversity Sabbatical Fellowship from the Ford Foundation. Work at Boston University was supported by CISM which is funded by the STC Program of the National Science Foundation under Agreement Number ATM-0120950. 


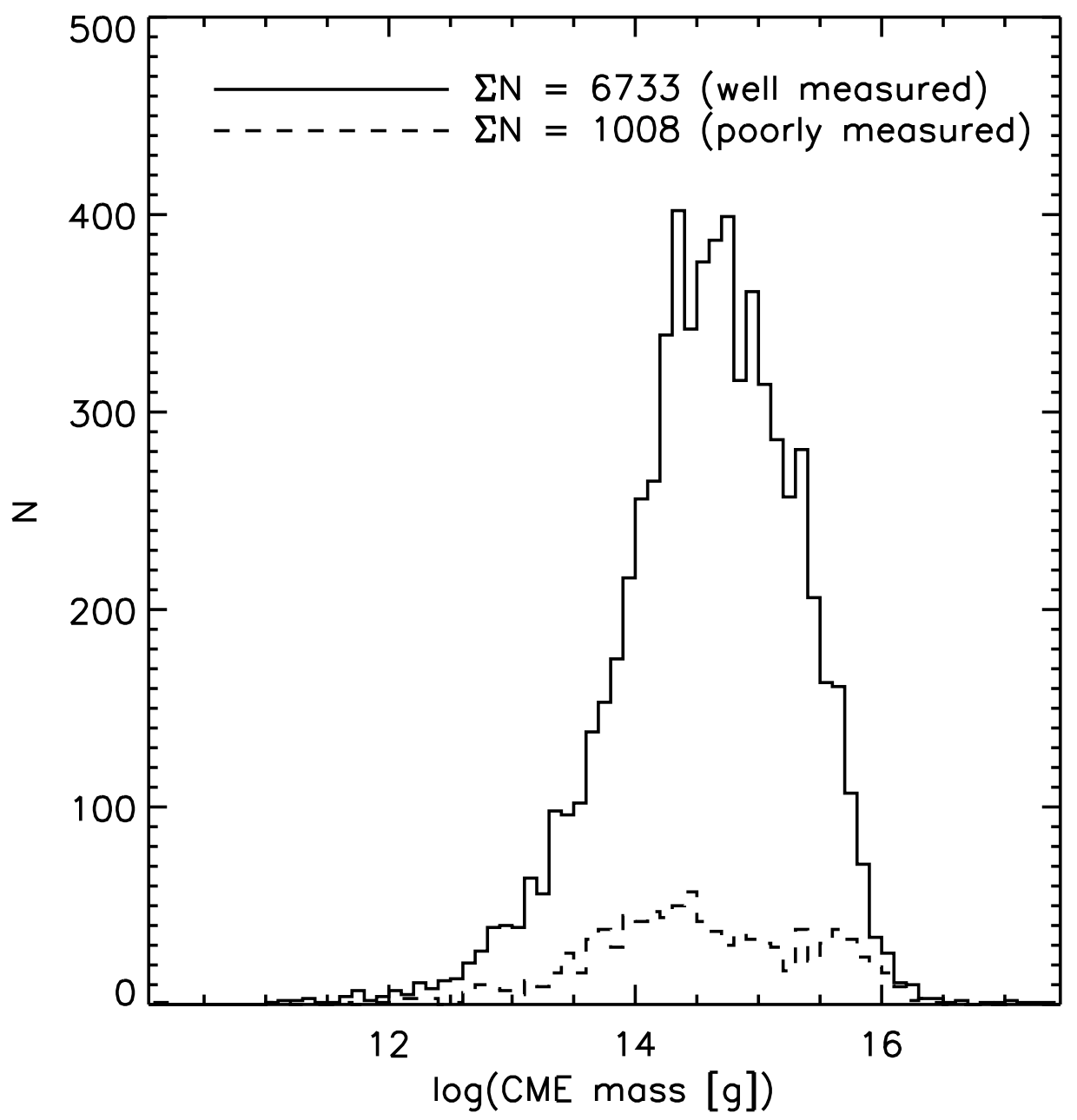

Figure 1. Summary of the LASCO CME database. From 1996 to 2006, there were 7741 CME mass measurements; 6733 were well-constrained, and 1008 were poorly constrained.

\section{References}

Andrews, M.D.: 2003, Solar Phys. 218, 261.

Andrews, M.D., Howard, R.A.: 2001, Space Sci. Rev. 95, 147.

Chen, J., Howard, R.A., Brueckner, G.E., Santoro, R., Krall, J., Paswaters, S.E., St. Cyr, O.C., Schwenn, R., Lamy, P., Simnett, G.M.: 1997, Astrophys. J. Lett. 490, L191.

Dryer, M.: 1996, Solar Phys. 169, 421.

Gopalswamy, N., Yashiro, S., Michalek, G., Stenborg, G., Vourlidas, A., Freeland, S., Howard, R.: 2009, Earth, Moon, Planets 104, 295.

Gosling, J.T., Hildner, E., MacQueen, R.M., Munro, R.H., Poland, A.I., Ross, C.L.: 1976, Solar Phys. 48, 389.

Haisch, B., Antunes, A., Schmitt, J.H.M.M.: 1995, Science 268, 1327.

Harrison, R.A.: 1991, Adv. Space Res. 11, 25. 


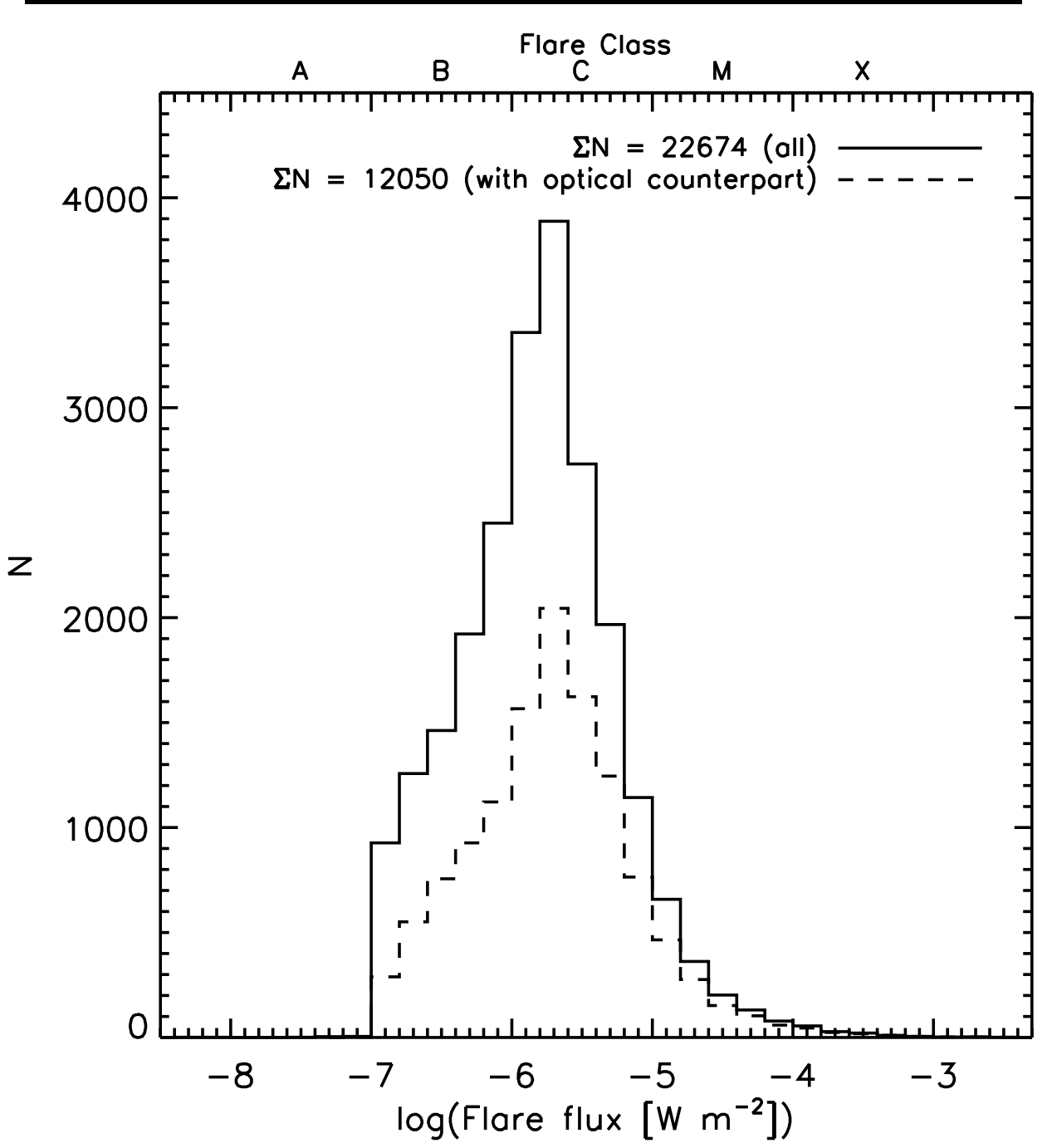

Figure 2. Summary of flare fluxes recorded in the GOES X-ray flare database from 1996-2006. 22674 flares in total were recorded, 12050 of which had measured positions of optical counterparts to the X-ray flare. For correlation with CMEs, we use only the 12050 flares with known positions.

Harrison, R.A.: 1995, Astron. Astrophys. 304, 585.

LaBonte, B.J., Georgoulis, M.K., Rust, D.M.: 2007, Astrophys. J. 671, 955.

Mahrous, A., Shaltout, M., Beheary, M.M., Mawad, R., Youssef, M.: 2009, Adv. Space Res. 43, 1032 .

Massi, M., Ros, E., Menten, K.M., Kaufman Bernadó, M., Torricelli-Ciamponi, G., Neidhöfer, J., Boden, A., Boboltz, D., Sargent, A., Torres, G.: 2008, Astron. Astrophys. 480, 489. Miura, H., Nakamoto, T.: 2007, Icarus 188, 246.

Moon, Y.J., Choe, G.S., Wang, H., Park, Y.D., Cheng, C.Z.: 2003, J. Korean Astron. Soc. 36, 61.

Munro, R.H., Gosling, J.T., Hildner, E., MacQueen, R.M., Poland, A.I., Ross, C.L.: 1979, Solar Phys. 61, 201. 


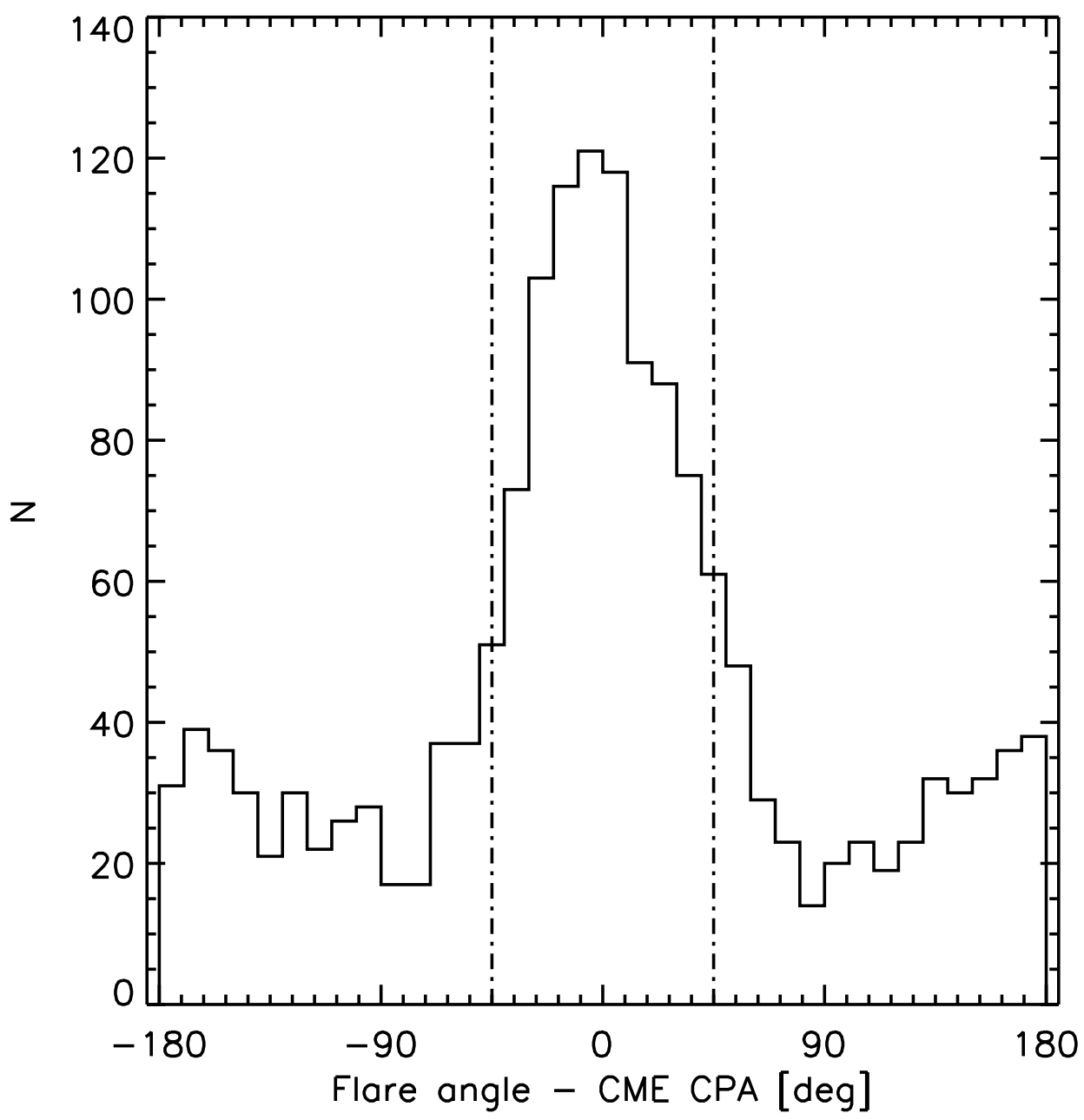

Figure 3. For flares and CMEs matched within $\mathrm{a} \pm 2 \mathrm{~h}$ time window, the angular separation of flares and CME central position angles shows a clear peak about $0^{\circ} \pm 45^{\circ}$ (vertical lines).

Nindos, A., Andrews, M.D.: 2004, Astrophys. J. Lett. 616, L175.

Peres, G., Orlando, S., Reale, F., Rosner, R.: 2001, Astrophys. J. 563, 1045.

Press, W.H., Flannery, B.P., Teukolsky, S.A., Vetterling, W.T.: 1995, Numerical Recipes in FORTRAN Example Book: The Art of Scientific Computing, Cambridge University Press, New York, NY, 692.

Reale, F., Betta, R., Peres, G., Serio, S., McTiernan, J.: 1997, Astron. Astrophys. 325, 782. Skelly, M.B., Unruh, Y.C., Cameron, A.C., Barnes, J.R., Donati, J.F., Lawson, W.A., Carter,

B.D.: 2008, Mon. Not. Roy. Astron. Soc. 385, 708.

St. Cyr, O.C., Webb, D.F.: 1991, Solar Phys. 136, 379.

Švestka, Z.: 2001, Space Sci. Rev. 95, 135.

Vourlidas, A., Subramanian, P., Dere, K.P., Howard, R.A.: 2000, Astrophys. J. 534, 456.

Webb, D.F., Hundhausen, A.J.: 1987, Solar Phys. 108, 383. 


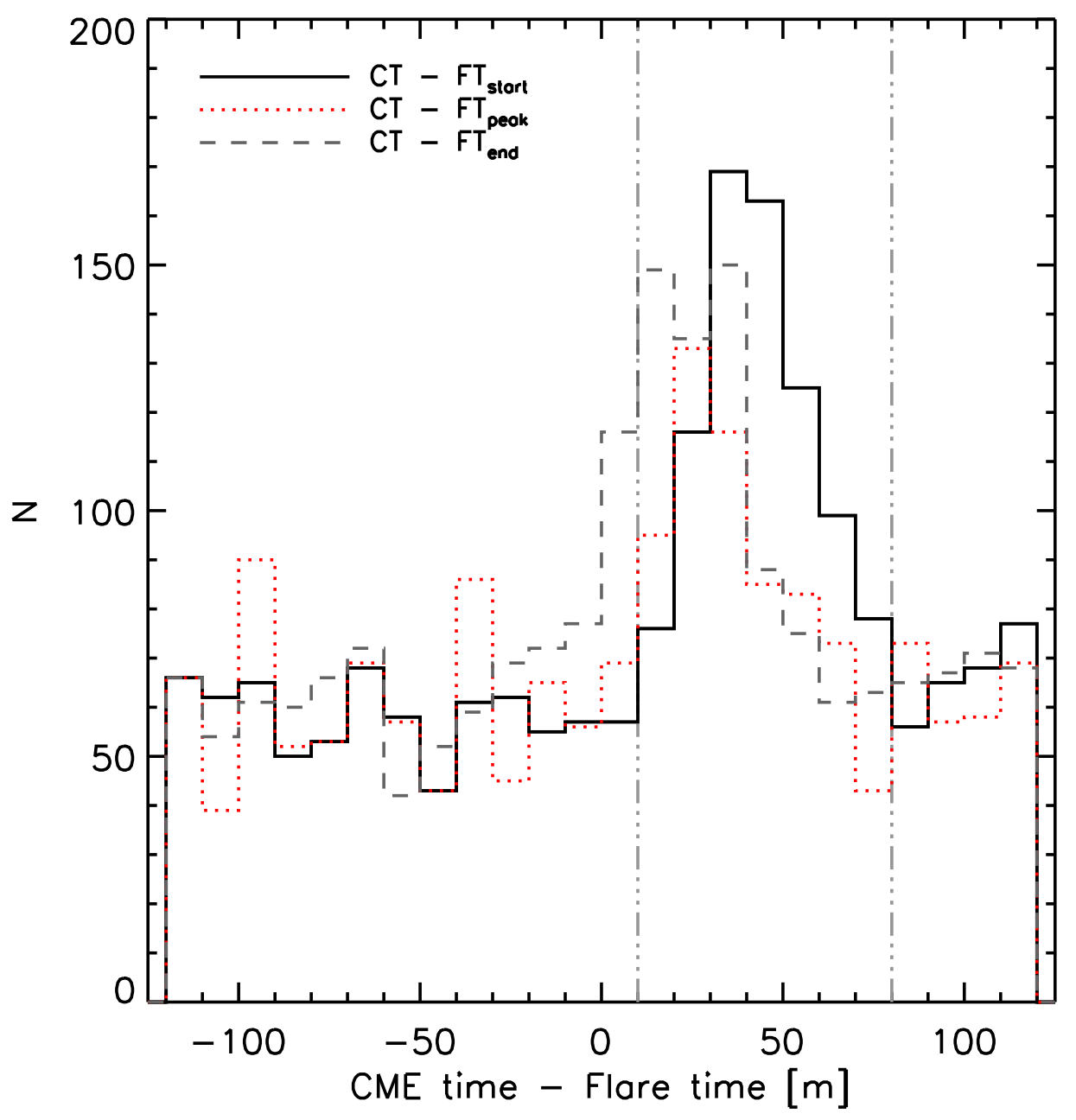

Figure 4. Assessment of which flare time to use for associating flares and CMEs; CT and FT refer to CME start time and flare time, respectively. We show here a $\pm 2 \mathrm{~h}$ time constraint as well as the $\pm 45^{\circ} \mathrm{CME}$ CPA-flare angle separation applied, the difference in each histogram being which flare time was used. Previous studies (e.g., Mahrous et al., 2009) found degree of flare-CME association to be dependent upon whether flare start, peak, or end time was used in flare-CME pairing. Time offsets are shown here of CME start time minus flare start (solid, black), peak (dashed, red), and end times (dot-dashed, gray). We observe the strongest peak in number of pairs when using the flare start time. The peak of the black histogram is a factor of $\approx 3$ above the "background" and well-defined in shape, spanning a range of time offsets from 10-80 min (triple dot-dashed, light gray vertical lines). 


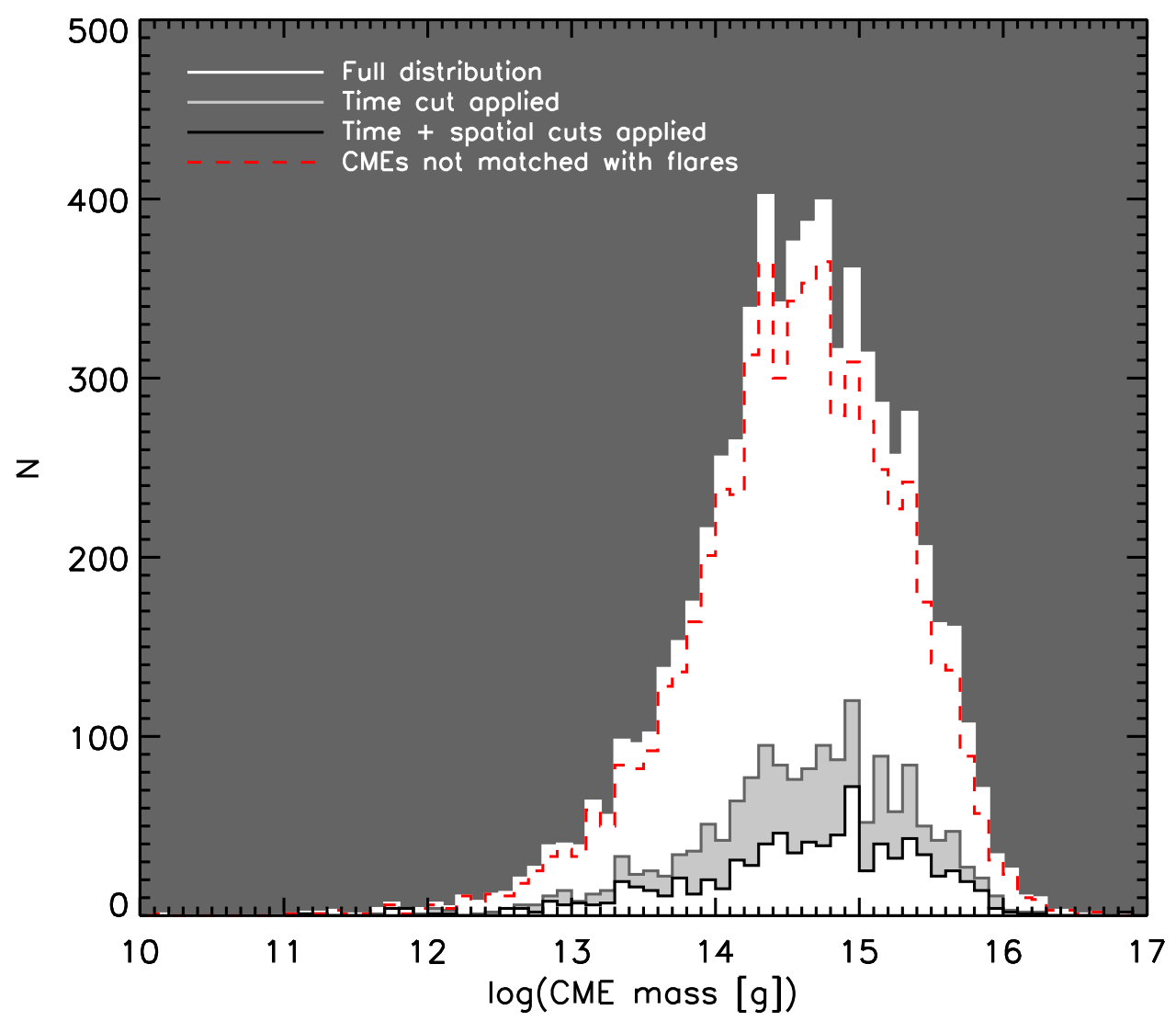

Figure 5. Change in the number and distribution of CME masses as flare/CME pairs are correlated. The white histogram bounded by dark gray shows the full initial distribution of well constrained CME mass measurements. Pairing flares with CMEs occurring 10-80 min after the flare start time, the data set is greatly reduced in number (filled, light gray histogram). The position criterion, $\mathrm{CME}$ and flare position angle equality within $\pm 45^{\circ}$, leaves 826 flare/CME pairs (solid black line, white filled histogram). In red, we show CMEs not associated with flares by these criteria.

Wood, B.E., Karovska, M., Chen, J., Brueckner, G.E., Cook, J.W., Howard, R.A.: 1999, Astrophys. J. 512, 484

Yashiro, S., Michalek, G., Akiyama, S., Gopalswamy, N., Howard, R.A.: 2008, Astrophys. J. 673, 1174 . 


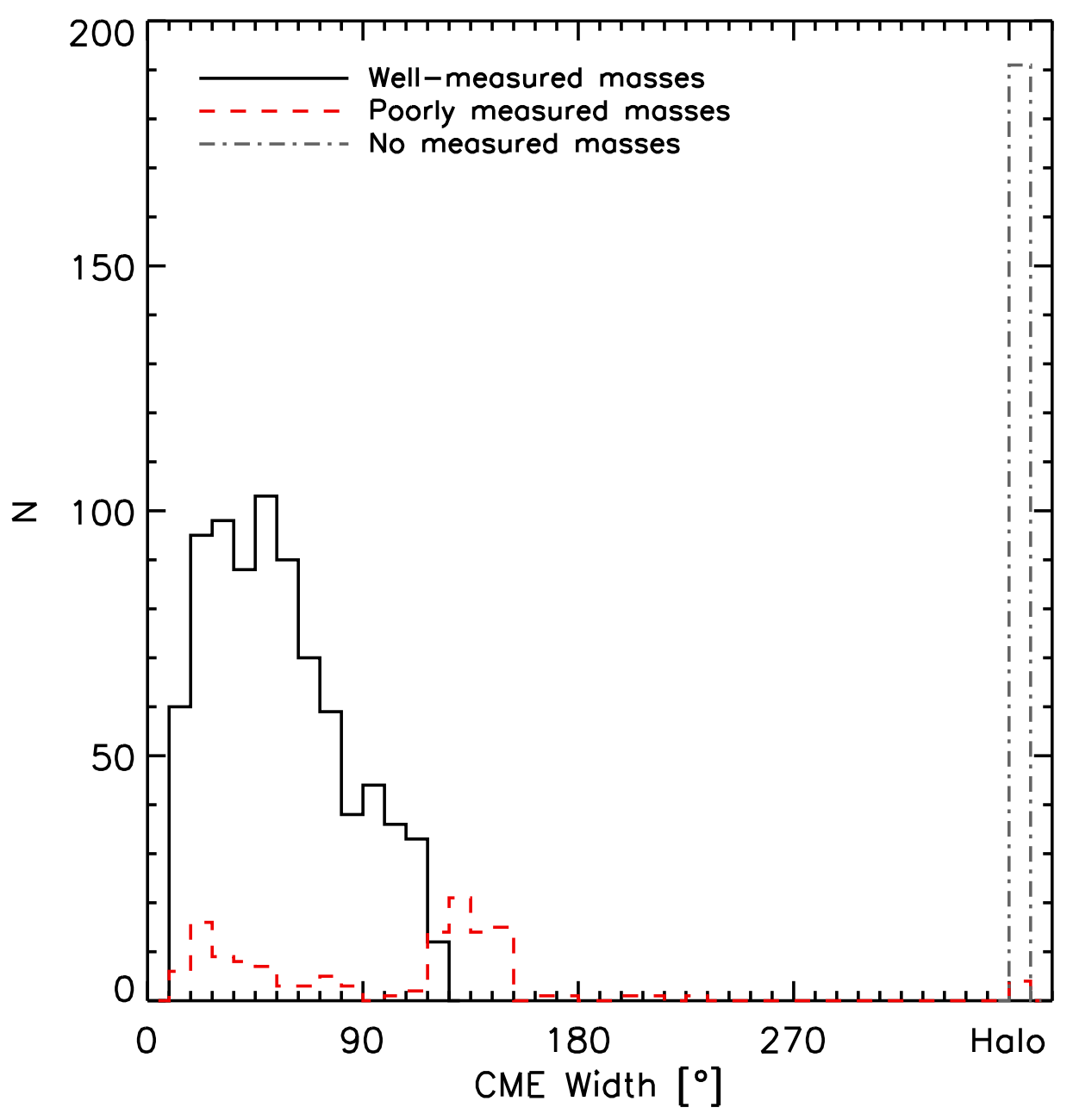

Figure 6. CME widths and frequency of "halo" CMEs. The distributions of widths for CMEs with well measured masses (solid, black line) and poorly constrained masses (dashed, red line) are shown. Masses are more difficult to measure, and thus the most poorly constrained, for the widest CMEs. All but three halo CMEs in our sample have no measured masses (dot-dashed, gray line). 


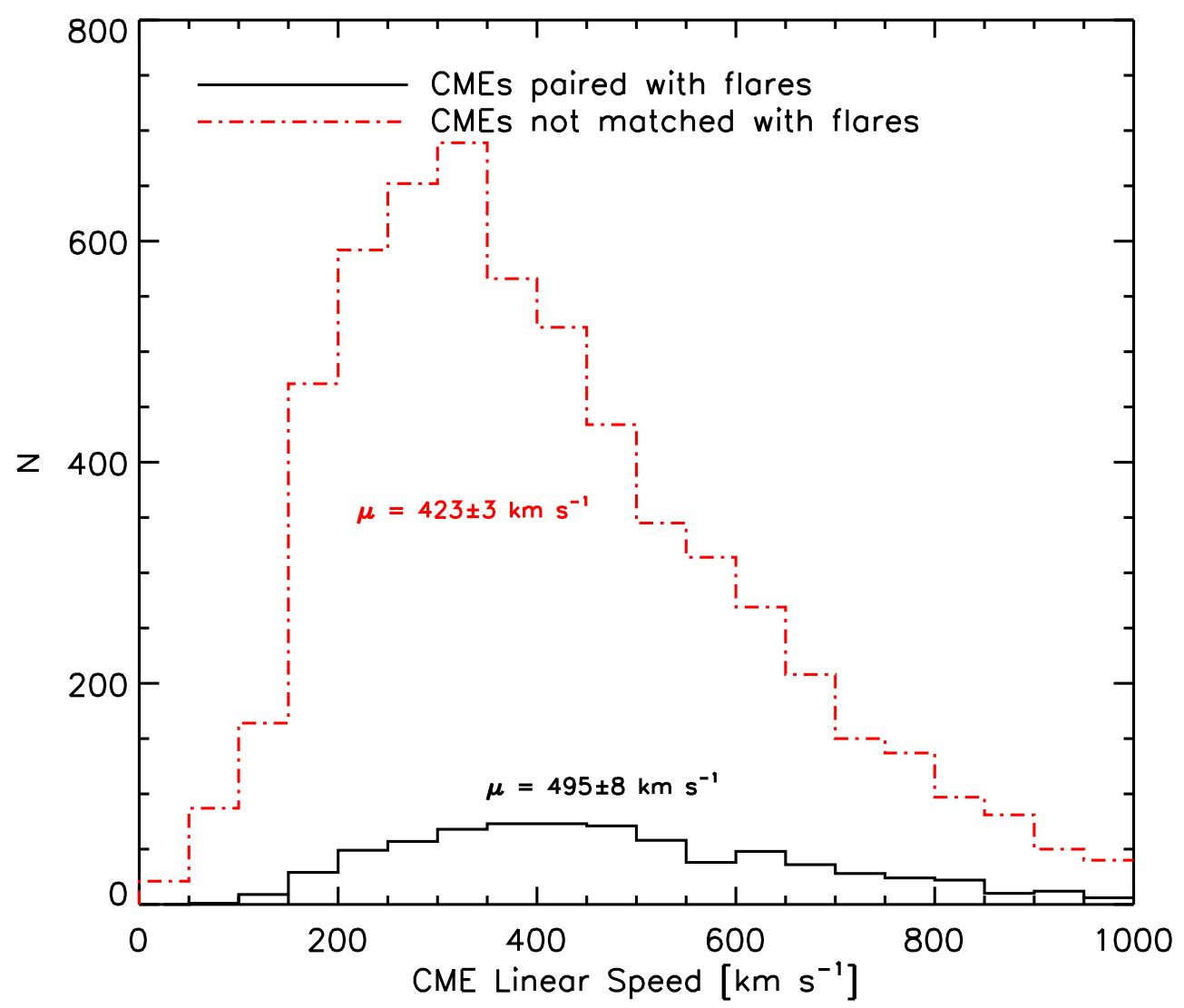

Figure 7. As in Figure 5 the red and black distributions show CMEs not matched with flares and CME/flare pairs, respectively. We find that CMEs associated with flares have, on average, faster linear speeds (see Section 4.2.1). In this and subsequent figures, we report on the plot the mean of each distribution with the error on that mean (i.e. the standard deviation of the mean, $\sigma / \sqrt{ } \mathrm{N})$. 


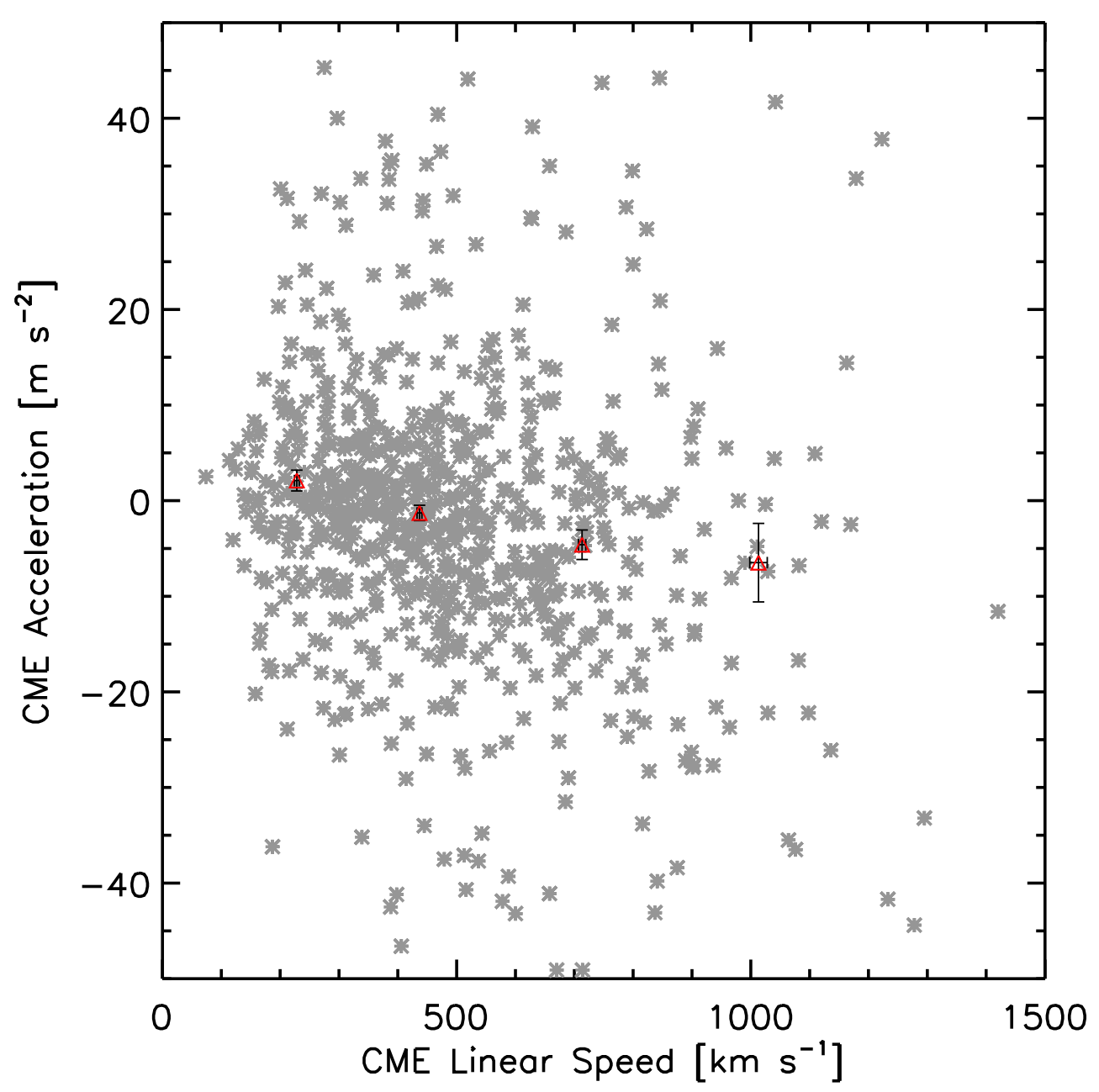

Figure 8. For flare-associated CMEs, linear speeds and accelerations are shown as gray asterisks. In bins $300 \mathrm{~km} \mathrm{~s}^{-1}$ wide in linear speed, mean speeds and accelerations are plotted as triangles (red). The figure has been cropped so the y-axis range of the triangles (red) can be clearly seen; 13 data points are not shown, lying beyond $\pm 50 \mathrm{~m} \mathrm{~s}^{-2}$. While the magnitude of the slope of these points is rather small, its existence is significant: a Spearman's $\rho$ correlation test shows these two quantities are highly significantly anticorrelated ( $\rho=-0.22$, null-hypothesis probability $\left.\ll 10^{-6}\right)$. We see here that the fastest CMEs tend to decelerate, while the slower CMEs accelerate through the C2 field of view (Section 4.2.1). 


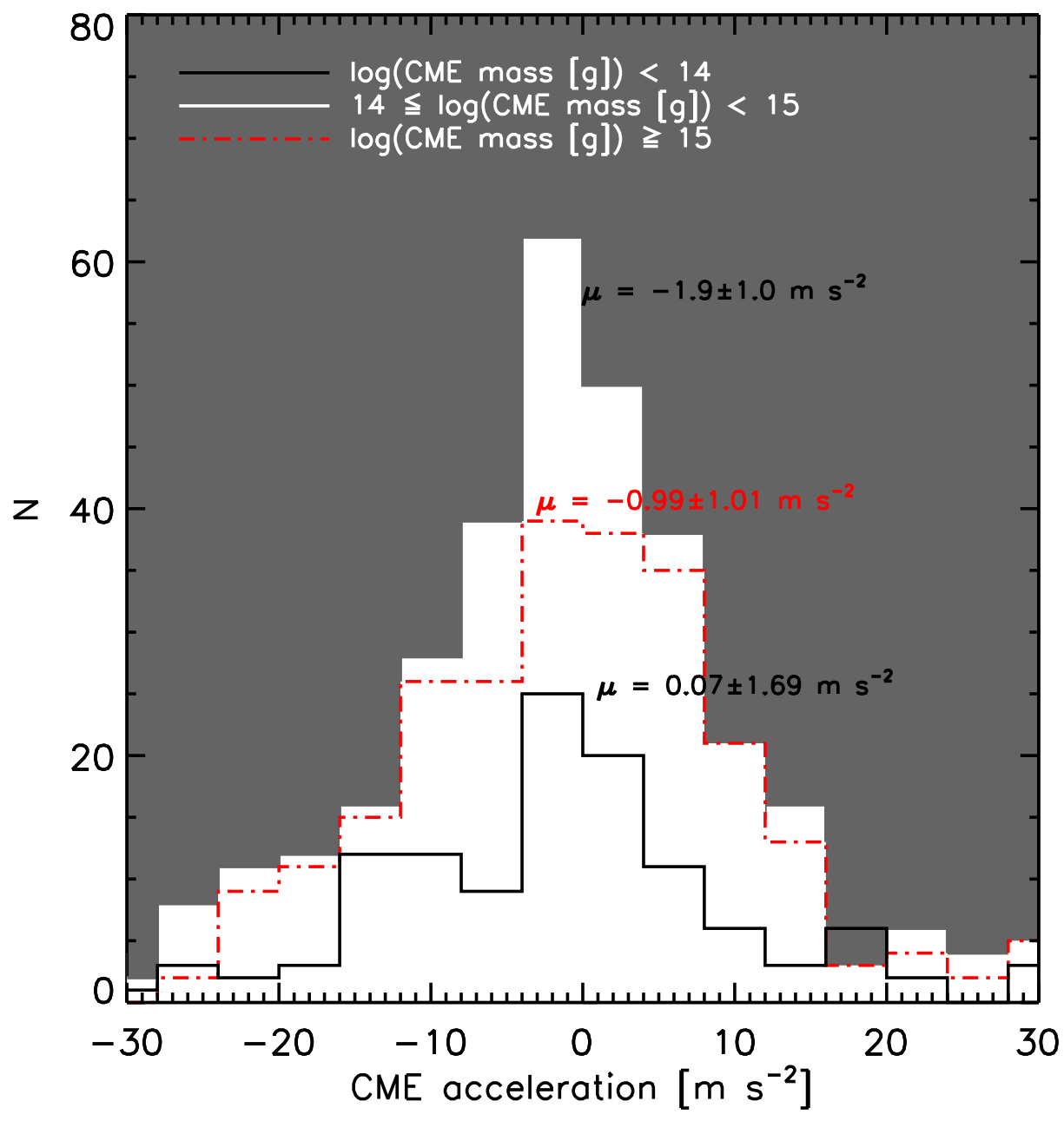

Figure 9. Relationship between CME mass and CME acceleration. The dot-dashed (red) histogram represents accelerations for the highest mass CMEs, with $\log (\mathrm{CME}$ mass $[\mathrm{g}]) \geq$ 15. The white histogram bounded by dark gray shows accelerations for CMEs with masses $>$ $10^{14} \mathrm{~g}$ and $<10^{15} \mathrm{~g}$. The lowest masses shown, $<10^{14} \mathrm{~g}$, are represented by the solid (black) outlined histogram. There is no apparent dependence of CME acceleration on CME mass (see Section 4.2.2. 


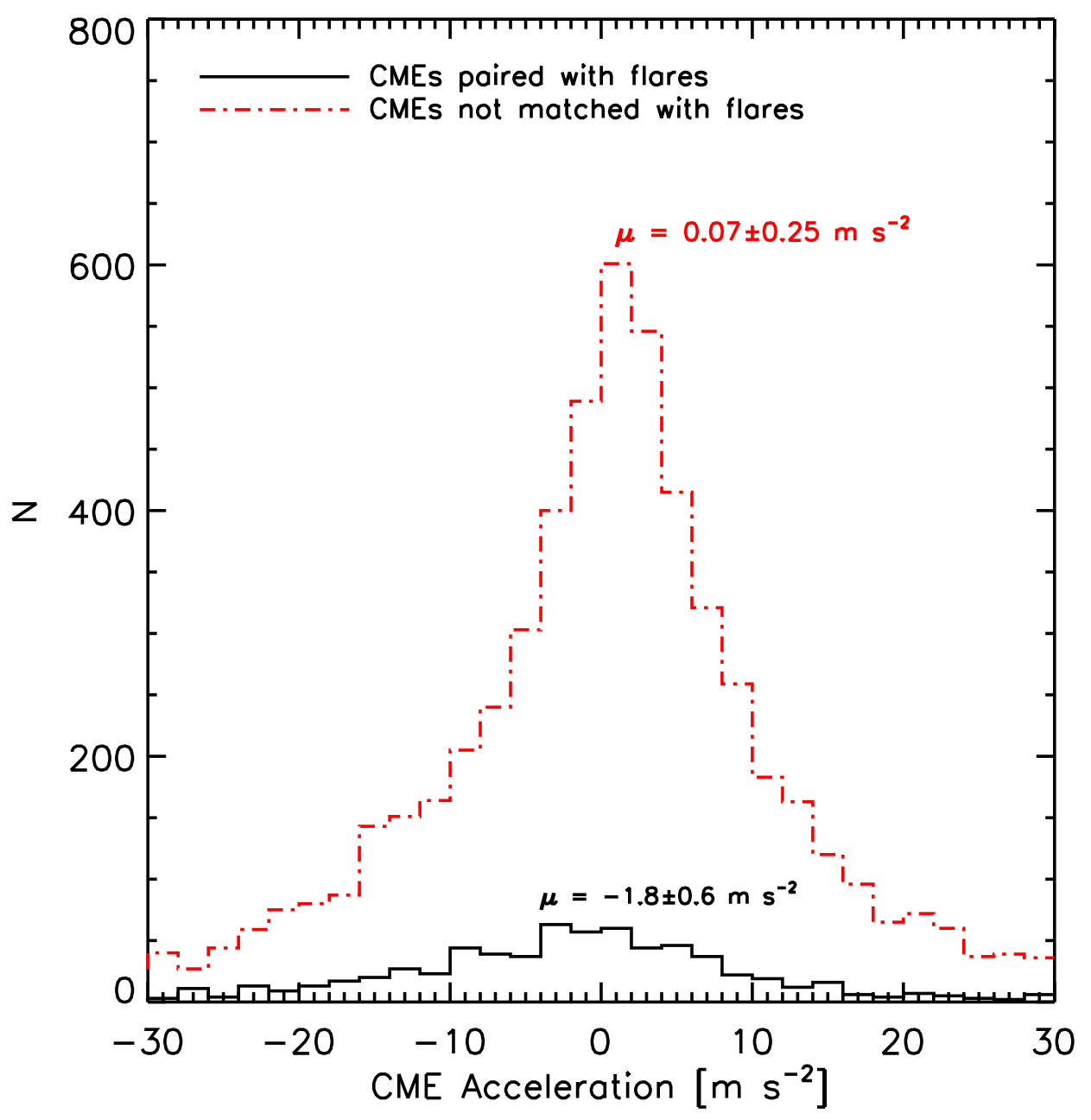

Figure 10. CME accelerations: CME/flare pairs (solid, black distribution) and CMEs not associated with flares (dot-dashed, red). The means of the two distributions (printed on the plot, with the associated standard deviations of each mean) are similar in magnitude but significantly different. The $t$ statistic is -2.5 , with a null-hypothesis probability of 0.01 . A non-parametric $U$ test confirms this, with a $Z$ statistic of 4.6 and a null-hypothesis probability of $2 \times 10^{-6}$ (see Section 4.2.2). 


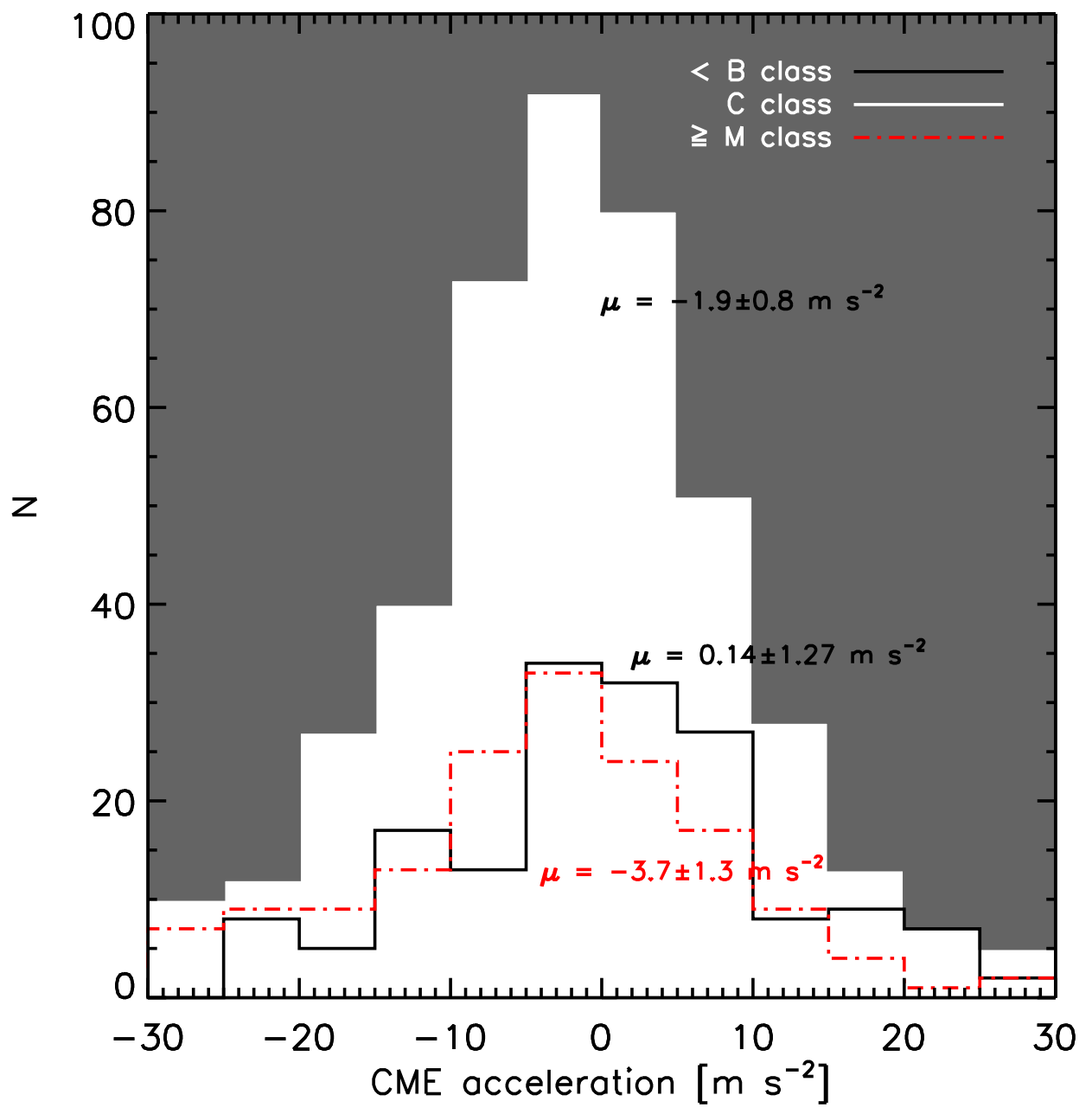

Figure 11. CME acceleration binned by flare flux. $\mathrm{M}$ and $\mathrm{X}$ class flares $\left(\log \left(\mathrm{flux}\left[\mathrm{W} \mathrm{m}^{-2}\right]\right) \geq\right.$ -5 ; combined here due to small number of X class flares in sample) are paired with CMEs that have accelerations as shown by the dot-dashed (red) distribution. C class flares $(-6 \leq \log$ (flux $\left.\left.\left[\mathrm{W} \mathrm{m}^{-2}\right]\right)<-5\right)$ have corresponding $\mathrm{CME}$ accelerations as shown by the white histogram bounded by dark gray. A and B class $\left(\log \left(\right.\right.$ flux $\left.\left.\left[\mathrm{W} \mathrm{m}^{-2}\right]\right)<-6\right)$ flares' associated CMEs have accelerations as shown by the solid (black) histogram. We report the mean value of each distribution, with the standard deviation of each mean, on the figure. The $\mathrm{B}$ class flare/CME acceleration distribution has a mean significantly different than the higher energy flare associated CMEs; CMEs associated with lower energy flares appear to modestly accelerate, while those associated with higher energy flares decelerate (see Section 4.2.2). 


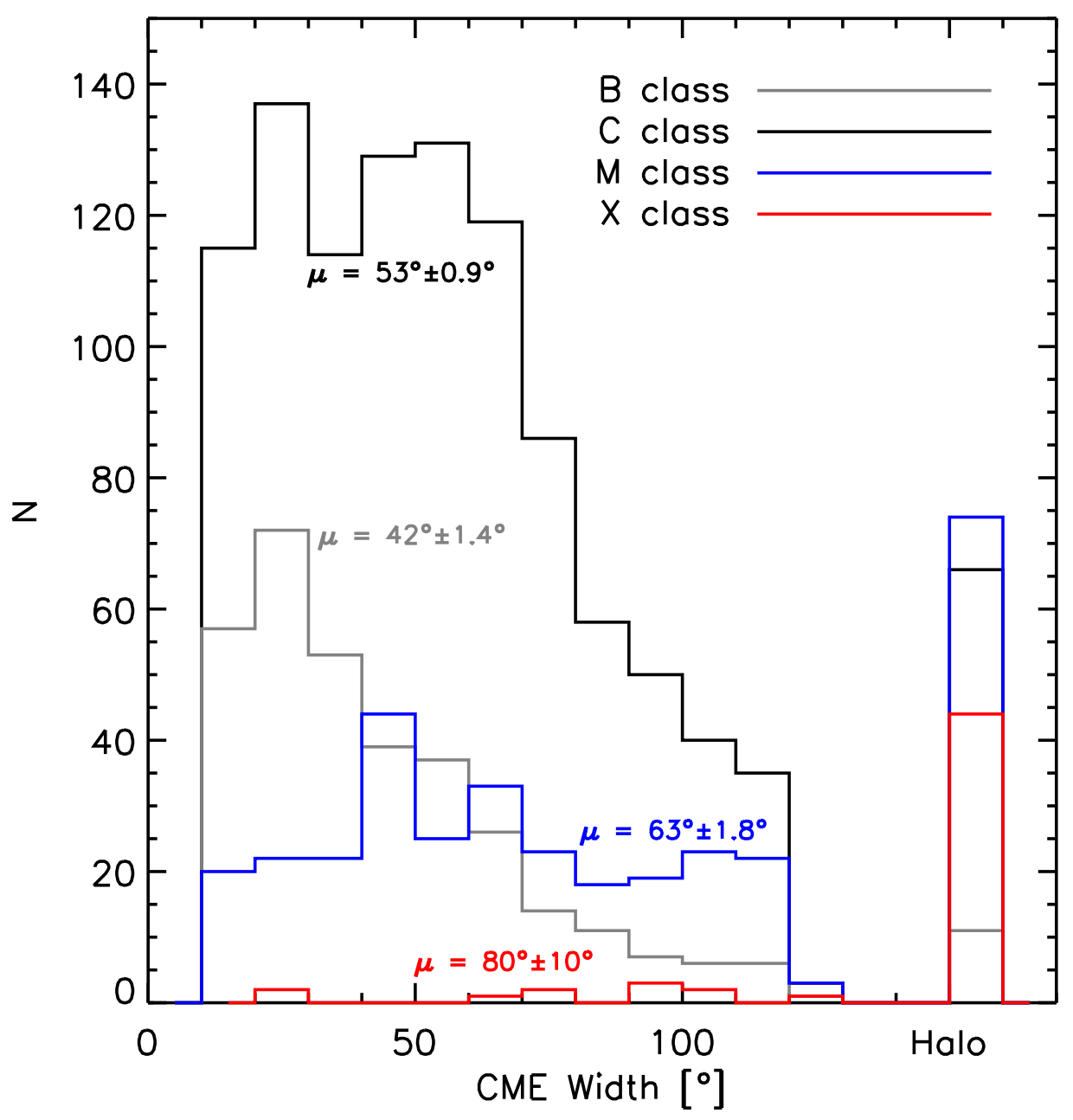

Figure 12. Widths of CMEs associated with flares of a given flux. The mean widths for each flare class are printed on the plot with the standard deviations of each mean. The mean widths for CMEs associated with M, C, and B class flares are statistically significantly different from one another. Interestingly, $80 \%$ of CMEs associated with X class flares have "halo" designations, that percentage decreasing with decreasing flare flux (see Section 4.2.3). 


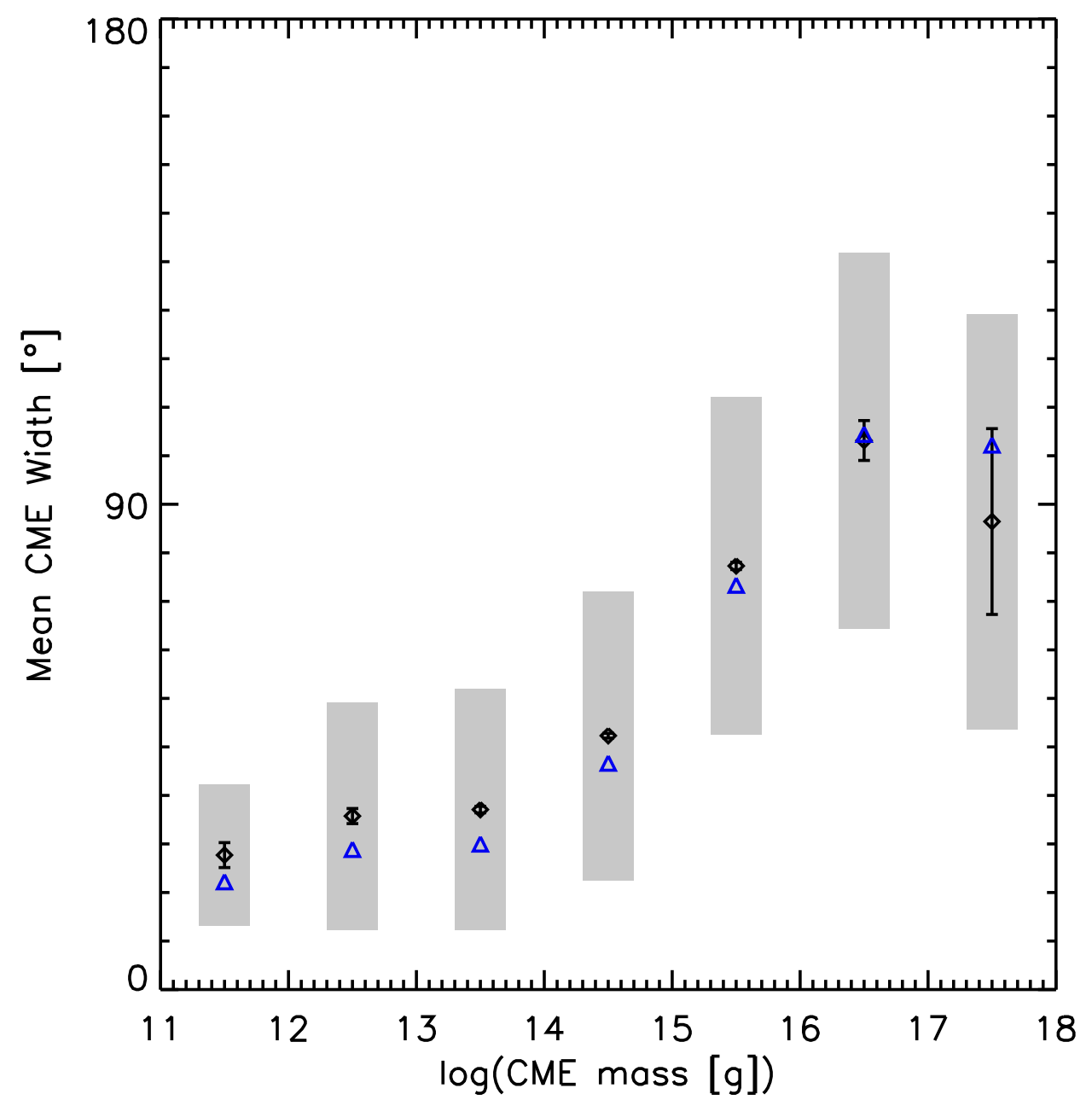

Figure 13. CME width and mass. Each point represents a binned order of magnitude in mass, each abscissa value plotted at the bin's center. The diamonds (black) are the mean CME width for each mass bin, and triangles (blue) are median values. Error bars show the error on each mean value, while the gray shaded boxes show the standard deviation of each mean. As CME width increases, so does the mass of the CME (see Section 4.2.3). 


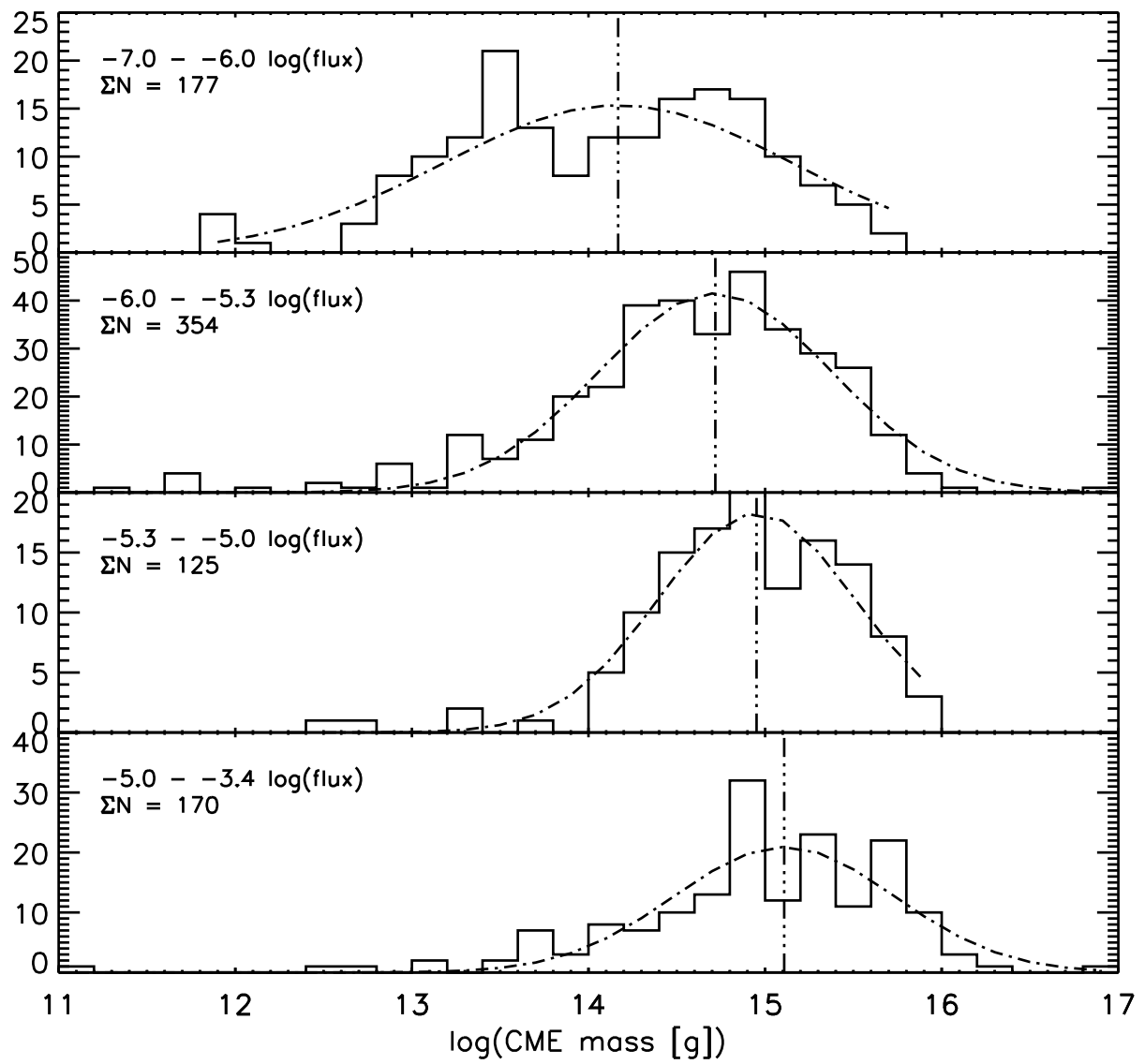

Figure 14. Grouping flare-CME pairs by flare flux, ascending flux from top to bottom, we see a clear increase in the centroid value of CME mass (Section 4.2.4). 


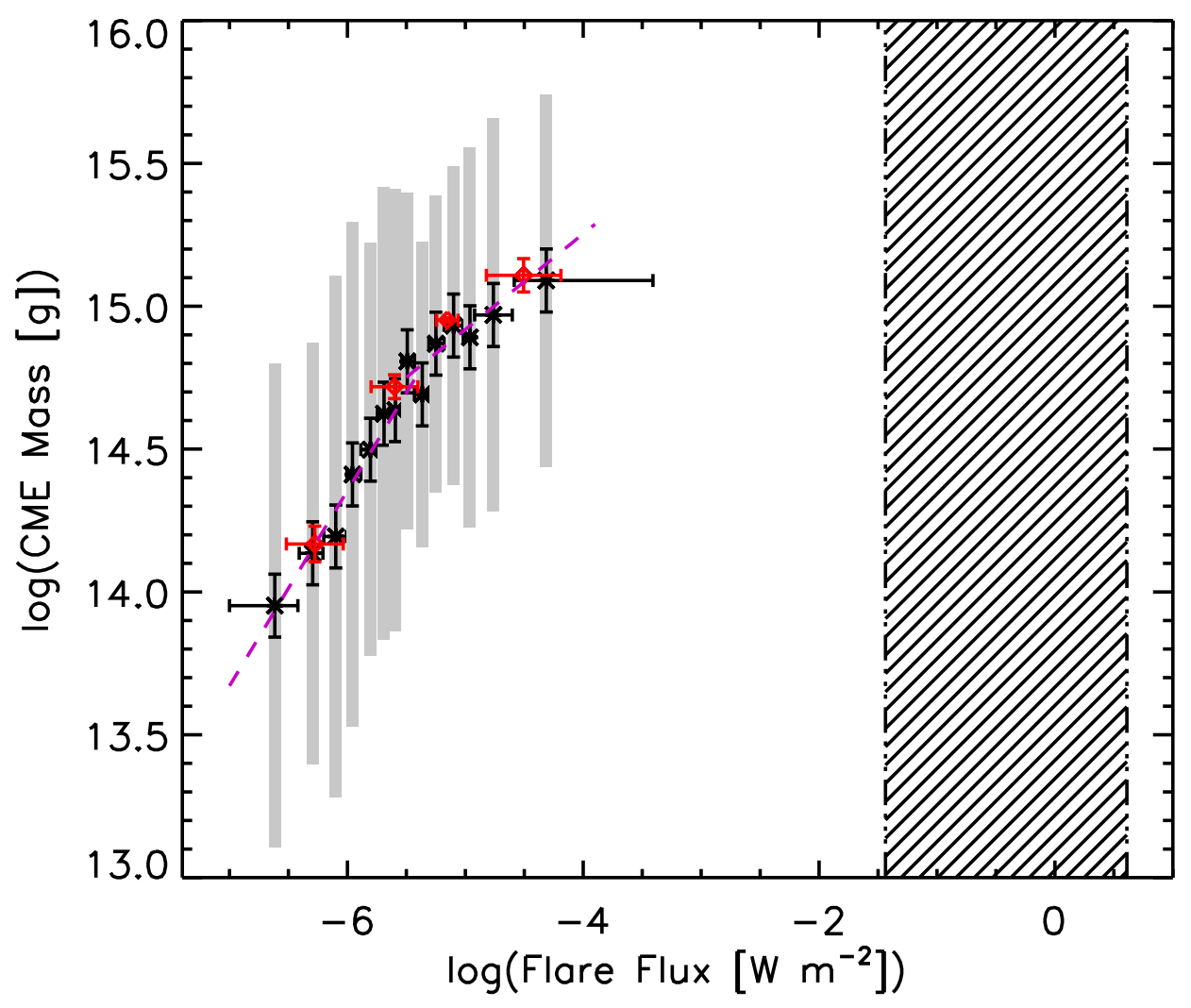

Figure 15. Relationship between CME mass and flare flux. Black exes: the 826 flare/CME pairs are binned into equivalent boxes of $N=59$. Each asterisk point is centered on the mean flux and mass per 59 pair bin. The flux error bars shows the minimum/maximum flare flux values spanned by that bin, and the mass error bars are the error on the mean (i.e., $\sigma / \sqrt{N}$ ). Four red diamonds: abscissae are the mean flux values for each of the groups as set in Figure $14 \pm \mathrm{x}$ error is the standard deviation of that mean. The ordinates are the mass values corresponding to the peaks of the Gaussian fits in Figure 14 their errors are the fit errors of the centroid. The light gray shaded boxes in the background are of arbitrary width, but show the standard deviation of the mean mass plotted in the foreground. For comparison, the hatched region represents the highest flux flares observed on young stars (see Section 1). Two linear functions are fit to the data, the first to bins $0-8$, and the second to bins $7-13$. There is slight overlap in that precisely where the "knee" of the function occurs is unclear. The first linear fit is of the form $\log (\mathrm{CME}$ mass $)=(18.5 \pm 0.57)+(0.68 \pm 0.10) \times \log$ (flare flux $)$. The second linear fit follows the form $\log (\mathrm{CME}$ mass $)=(16.6 \pm 1.30)+(0.33 \pm 0.26) \times \log$ (flare flux $)$. 


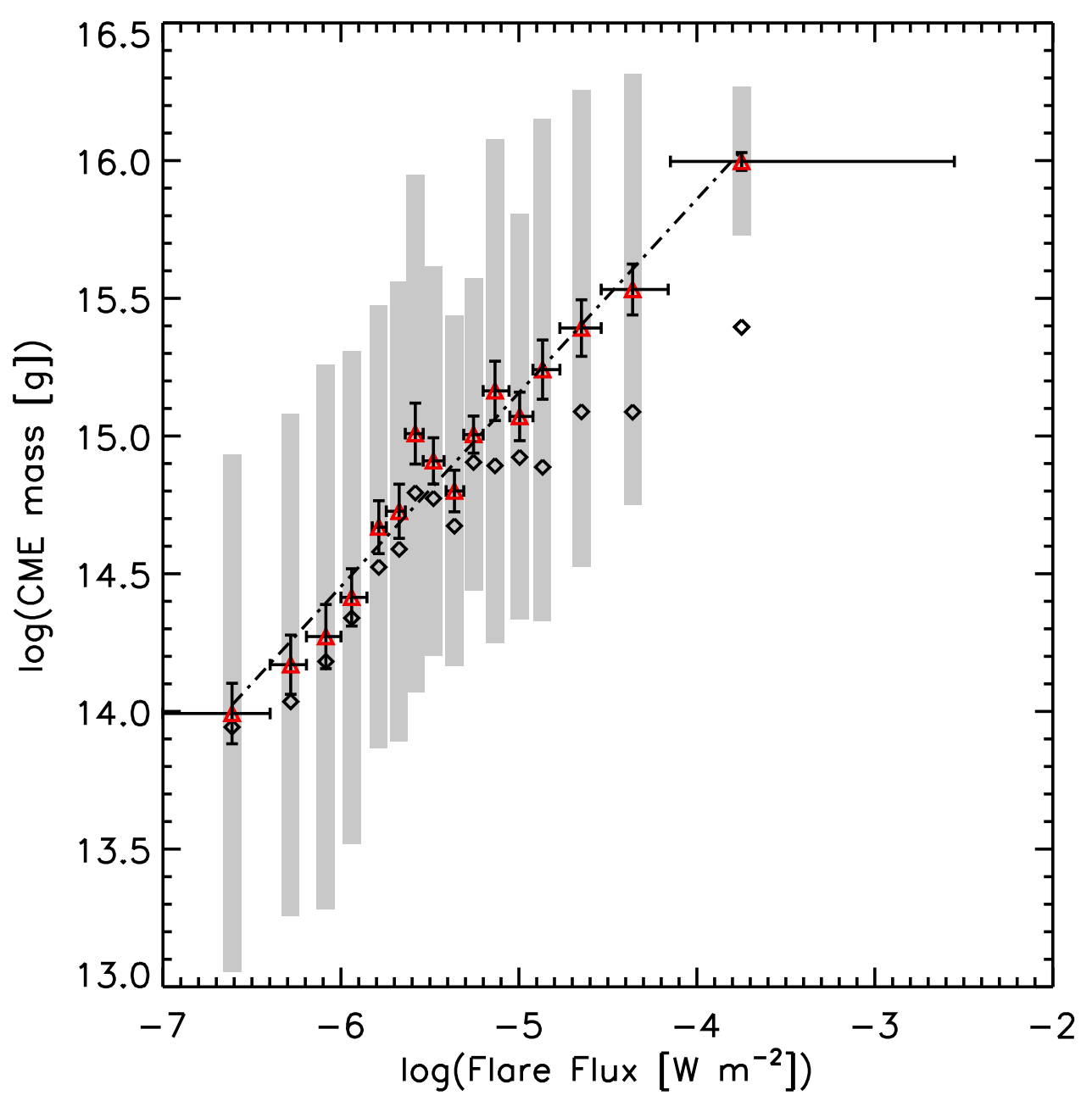

Figure 16. As in Figure 15, the relationship between flare flux and CME mass is shown. In this case, we include the sample of CMEs with poorly constrained mass measurements (Figure 1] dashed histogram), and see a relationship (diamonds, black) much like the one in Figure 15 The triangles (red) indicate how the function would change were we to include halo CMEs; we assign halo CMEs the maximum mass from the bin occupied by its associated flare. This function, fit by the dot-dashed (black) line, represents the steepest slope we could anticipate assuming halo CMEs have masses which lie within the observed distribution. 